"This is the peer reviewed version of the following article: New alkene cyclopropanation reactions enabled by photoredox catalysis via radical carbenoids which has been published in final form at DOI: 10.1055/s-00371611872. This article may be used for non-commercial purposes in accordance with Thieme Terms and Conditions for Self-Archiving".

\title{
New alkene cyclopropanation reactions enabled by photoredox catalysis via radical carbenoids
}
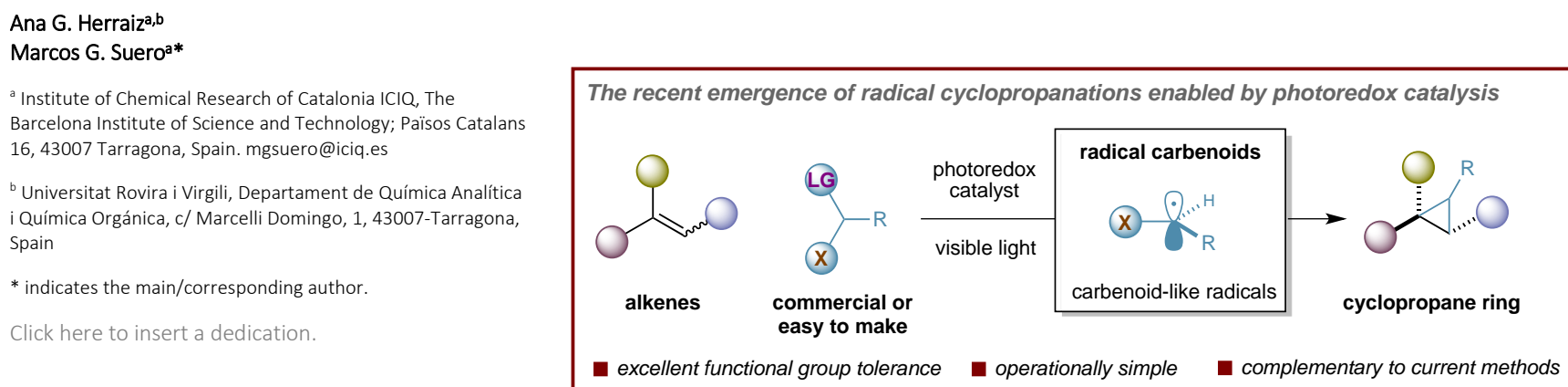

Received:
Accepted:
Published online:
DOI:
Abstract
We describe the recent emergence of a new approach for the synthesis of
cyclopropane rings by means of photoredox catalysis. This methodology relies
on the photocatalytic generation of radical carbenoids or carbenoid-like
radicals as cyclopropanating species, and its characterized by an excellent
functional group tolerance, chemoselectivity and ability to cyclopropane E/Z
alkene mixtures with excellent stereocontrol. The mild reaction conditions and
the employ of user-friendly reagents are highly attractive features that may
find immediate use in academic and industrial laboratories.
Table of Contents
1. Introduction
2. Photoredox-catalyzed alkene cyclopropanations with radical
carbenoids
3. Conclusions and outlook

Key words photoredox catalysis, radicals, carbenoids, cyclopropanes, cyclopropanation

\section{Introduction}

The cyclopropane ring is an important carbon structure class present in a large variety of natural products and medicines as well as in agrochemicals and perfumes (Scheme 1). ${ }^{1}$ This threemembered ring is also a versatile building block in organic synthesis; its innate ring strain and "double bond character" has permitted the development of a wide plethora of reactions such as ring-openings, cycloadditions and rearrangements. ${ }^{2}$ Cyclopropanes are also recognized as privilege scaffolds in drug discovery because (i) it is an alkyl bioisostere that improves metabolic stability compared to other alkyl groups and (ii) it
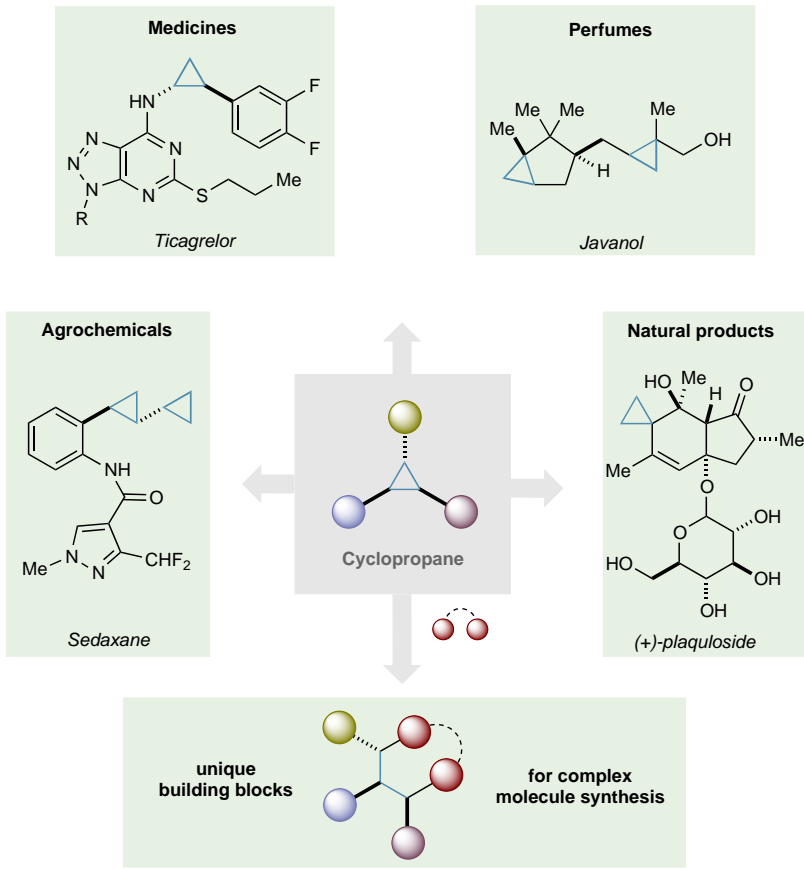

provides a conformationally restricted distribution of the substituents. ${ }^{3}$

Scheme 1 Prevalence of cyclopropane rings

Nature constructs cyclopropane rings through biosynthetic pathways that often involves ring-closing events with alkenes and carbocations. ${ }^{4}$ Synthetic chemists also employ alkenes as 
substrates in cyclopropane construction. The most common strategies rely on metal-carbenoids, metal-carbenes \& free carbenes, as well as sulfur and nitrogen ylides as divalent carbon sources (Scheme 2A). ${ }^{5}$ With only these three major strategies, chemists have literally delivered thousands of alkene cyclopropanation reactions. The main challenge over the years has been to develop general catalytic methodologies for the diastereo- and enantioselective cyclopropane synthesis under mild conditions, avoiding the use of highly toxic or explosive precursors. ${ }^{6}$ These three main methodologies, among others, have extensively been highlighted over the years in many reviews, and what is remarkable is to find the absence of methodologies involving radical species able to cyclopropanate alkenes.

The aim of this short review is to highlight the recent emerge of radical carbenoids or carbenoid-like radicals as novel reactive species for alkene cyclopropanation enabled by photoredox catalysis (Scheme 2B). These carbon-centered radicals have the particular feature of bearing a halogen atom $(\mathrm{X})$ in alpha position and can be generated via single-electron transfer process from photoreducible or photooxidable sources. These radical species are able to attack olefins with an excellent selectivity profile and form new radicals that evolve to the corresponding cyclopropane ring through two well-distinguished pathways (i) radical $\left(\mathrm{S}_{\mathrm{H}} 2\right) 3-$ exo-tet cyclization or (ii) single-electron reduction/anionic $\left(\mathrm{S}_{\mathrm{N}} 2\right)$ 3-exo-tet cyclization. The common and distinguished features as well as the proposed mechanisms of the presented individual works are highlighted in this review.

A Classic reactive species in alkene cyclopropanation
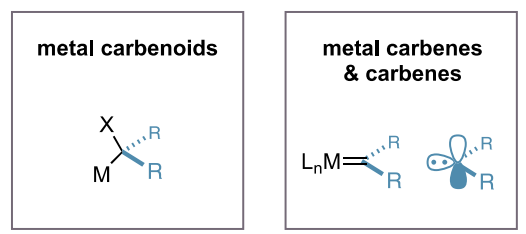

S \& $N$ ylides

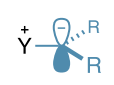

B Photoredox-catalyzed cyclopropanation reactions with radical carbenoids

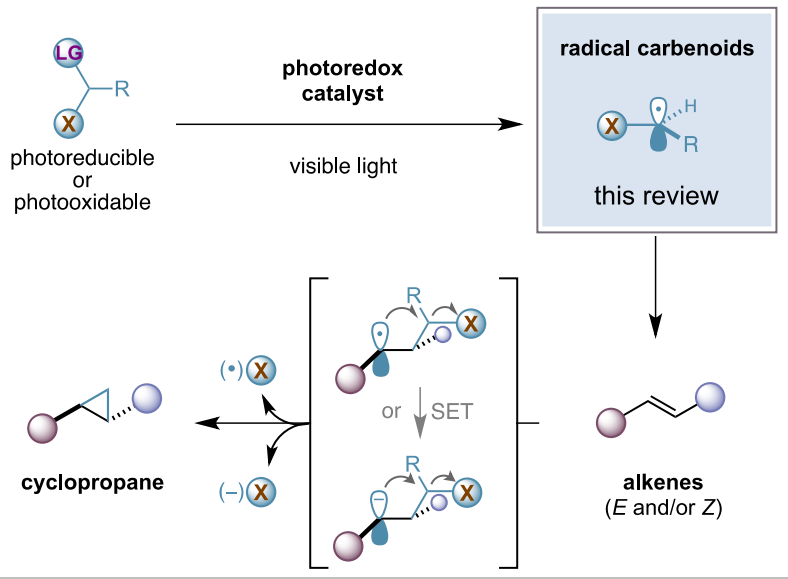

Scheme 2 Reactive species in cyclopropane synthesis and new radical methodologies enabled by visible-light photoredox catalysis.

\section{Photoredox-catalyzed alkene cyclopropanations with radical carbenoids}

Visible-light photoredox catalysis has emerged as a new smallmolecule activation mode..$^{7}$ In a general sense, this approach is based on the activation of organic molecules through singleelectron/energy transfer processes with metal complexes or organic dyes. The catalyst absorbs light in the visible region of the electromagnetic spectrum to give long-lived photo-excited states, which have the remarkable properties of being both more oxidizing and more reducing than the ground-state species. One of the most important features of this methodology is the ability to generate radical cation or anion species that evolve into transient radical species.

The groups of Macmillan and Stephenson, in 2008 and 2009, respectively, demonstrated the ability of photoredox catalysis to enable generation of carbon-centered radicals from alkyl halides using visible-light irradiation. ${ }^{8}$ These works clearly demonstrated that visible-light photoredox catalysis was a more efficient alternative in the generation of radical species than classic methods relying on photoinduced electron transfer with UV-light, ${ }^{9}$ or in the use of stoichiometric amounts of toxic reagents, such as tributyltin hydride. ${ }^{10}$

Inspired by the works of Macmillan and Stephenson, our group recognized the suitability of photoredox catalysis to enable a general platform for methylene transfer to alkenes using commercially available diiodomethane $\left(\mathrm{CH}_{2} \mathrm{I}_{2}\right) \cdot{ }^{11}$ It has an accessible reduction potential $\left(E_{\text {red }}=-1.44 \mathrm{~V}\right.$ vs. SCE $)$ with common photoredox catalysts and importantly, does not share the intrinsic drawbacks of other classic methylene sources (high oxygen and moisture sensitivity, explosiveness and toxicity) such as halomethyl organometallics (i.e. $\mathrm{ICH}_{2} \mathrm{ZnI}$ ) or diazomethane. We hypothesized that photoredox catalysis would enable the generation of iodomethyl radical $(\bullet) \mathrm{CH}_{2} \mathrm{I}$ as radical carbenoid species. We anticipated that $(\bullet) \mathrm{CH}_{2} \mathrm{I}$ may behave as a novel triplet carbene equivalent able to form trans-cyclopropanes from $E, Z$ styrene mixtures in a stereoconvergent manner.

In 2017, we reported the first stereoconvergent cyclopropanation reaction of styrenes with diidomethane and $\mathrm{N}, \mathrm{N}$-diisopropylethylamine using the well-known photocatalyst $\left[\mathrm{Ru}(\mathrm{bpy})_{3}\right]\left[\mathrm{PF}_{6}\right]_{2} \mathbf{3}$ (Scheme 3). ${ }^{12}$ We demonstrated the utility of this new cyclopropanation reaction in a broad range of $\beta$ substituted styrenes $\mathbf{1}$ as $E, Z$ mixtures, decorated with diverse functionalities. A notable feature of this process is the excellent functional group tolerance. For instance, aldehydes (2a), tertiary amines (2f) or sulfides (2e) are typical functionalities not tolerated by classic methodologies. Moreover, we observed an excellent site-selectivity in styrenes functionalized with an inactivated alkene (2e) and absolute stereoconvergence for a $E, Z$ mixture of trisubstituted olefin (2f), which represent a rare example in alkene functionalization. Current limitations of this cyclopropanation are (i) low efficiency for styrenes substituted with electron-withdrawing groups, (ii) incompetence to cyclopropanate terminal and $\alpha$-substituted styrenes $(2 \mathbf{g ~ h})$, and (iii) inability of accessing the cis-cyclopropane isomer. To reach full conversion in the cyclopropanation was necessary the degasification of the reaction mixture prior to irradiation and the addition of $\mathrm{Na}_{2} \mathrm{~S}_{2} \mathrm{O}_{3}$ and water as additive and co-solvent, respectively. In analogy to most of the photoredox processes, our cyclopropanation is robust and operationally simple and in addition, it required a simple $21 \mathrm{~W}$ compact fluorescent lamp (CFL) as visible-light source.

In the proposed mechanism, visible-light irradiation of the $\mathrm{Ru}$ catalyst generates the long-lived photoexcited state 
${ }^{*}[\mathrm{Ru}(\mathrm{bpy} 3)]^{2+} \mathbf{4}$, which is reduced by $N, N$-diisopropylethylamine to $[\mathrm{Ru}(\mathrm{bpy} 3)]^{+} \mathbf{5}$ by a well-established single-electron transfer (SET) process. The strong reductant generated $\mathrm{Ru}(\mathrm{I}) \mathbf{5}\left(E_{\mathrm{red}}(\mathrm{II} / \mathrm{I})=-\right.$ $1.33 \mathrm{~V}$ vs. SCE), donates an electron to $\mathrm{CH}_{2} \mathrm{I}_{2}\left(E_{\text {red }}=-1.44 \mathrm{~V}\right.$ vs. SCE $)$ to form a transient radical anion that subsequently fragments into carbenoid $(\bullet) \mathrm{CH}_{2} \mathrm{I}$ 6. This SET is slightly endergonic, however, the overall process may be driven by an exergonic radical addition to the $E, Z$-alkene mixtures that conducts to intermediates $\mathbf{7}$ and $\mathbf{8}$, which might be in equilibrium through a $\mathrm{C}-\mathrm{C}$ bond rotation. The ring closing event occurs on intermediate $\mathbf{8}$ with the anti-orientation of the substituents, to yield the most stable trans-cyclopropane 2 . The ring-closing event involves a radical $\mathrm{S}_{\mathrm{H}} 2$-type 3-exo-tet cyclization; this homolytic substitution reaction that generates an $\mathrm{I}(\bullet)$ has been observed previously from 1,3-dihaloalkanes via 3-halo-propyl radicals. ${ }^{13}$ Control experiments clearly discarded isomerizations of both styrene starting materials or cyclopropane products under our reaction conditions.
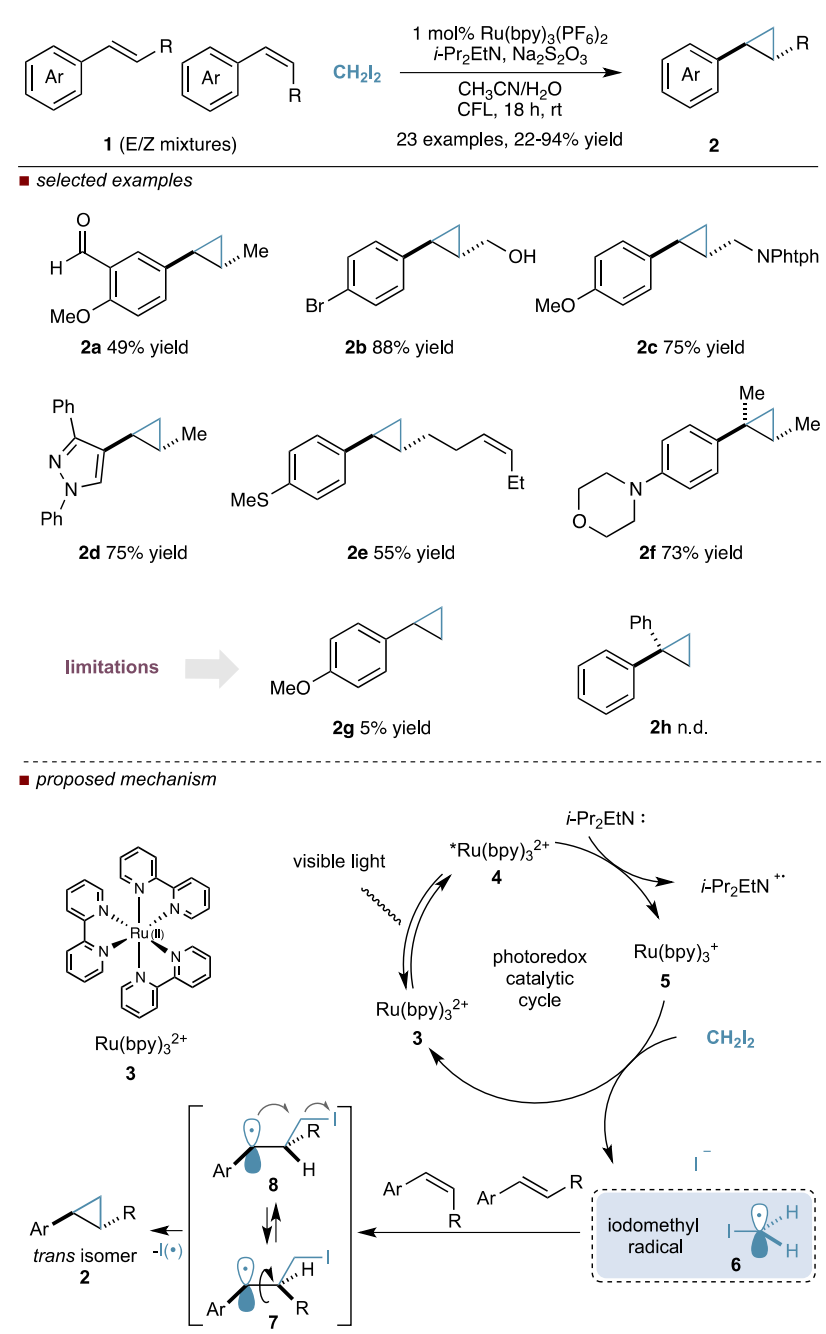

Scheme 3 Stereoconvergent cyclopropanation reaction enabled by photoredox catalysis with diiodomethane by Suero et. al.

Taking advantage of the reactivity of our radical carbenoid, our group later developed a photoredox-catalyzed cyclopropanation reaction of Michael acceptors 9 by using same reaction conditions previously developed (Scheme 4). ${ }^{14}$ We demonstrated this process in a wide range of substrates, including chalcones bearing electron-rich and electron-poor aromatic rings, heterocycles (10a), as well as $\alpha, \beta$-unsaturated aldehydes (10c) and ketones with moderate to excellent yields (36-93\%). Excellent site-selectivity was observed and only transcyclopropane products obtained when using isomeric E,Zmixtures of chalcones. Moreover, we demonstrated that more complex cyclopropane cores (10d) can be formed by using 1,1diiodoethane as a source of iodoethyl radical carbenoid 11. As limitation, we observed that $\alpha, \beta$-insaturated esters were no tolerated under our reaction conditions (10e). Previosly, the generation of iodomethyl radical species with $\mathrm{CH}_{2} \mathrm{I}_{2} / \mathrm{BEt}_{3} / \mathrm{O}_{2}$ and subsequent 1,4-addition to methyl vinyl ketone was reported. ${ }^{15}$ However, a Michael adduct was obtained and no cyclopropane was observed.

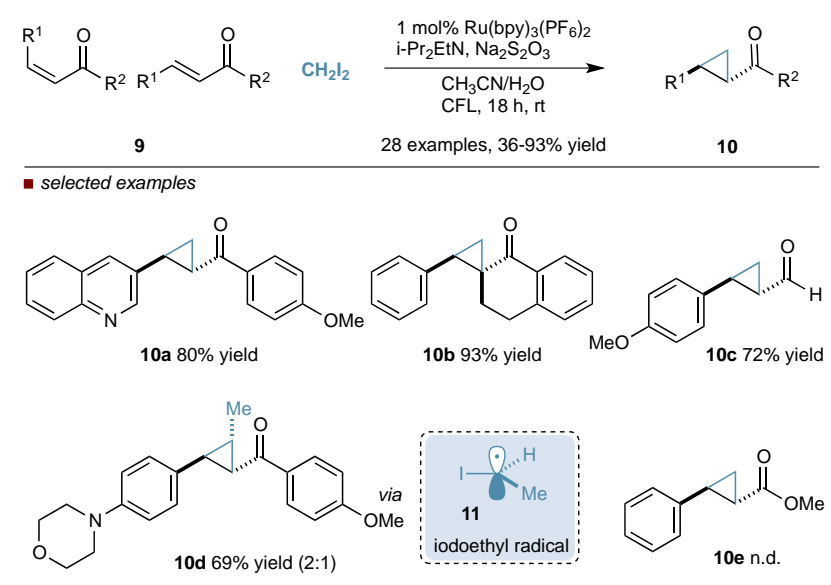

Scheme 4 Photoredox-catalyzed cyclopropanation reaction of Michael Acceptors by Suero et. al.

In 2018, the Molander group reported a redox-neutral photocatalytic cyclopropanation via radical/polar crossover (Scheme 5). ${ }^{16}$ A key part of this work was the design and synthesis of the benchtop stable triethylammonium bis(catecholato)iodomethylsilicate $\mathbf{1 3}$ as source of iodomethyl radical 6, made from commercial chloromethyltrimethoxysilane 12 and commodity chemicals in two steps. ${ }^{17}$ Using this reagent and 1,2,3,5-tetrakis(carbazol-9-yl)-4,6-dicyano-benzene (4CzIPN) 16 as photocatalyst, Molander and co-workers demonstrated cyclopropanations in a broad range of olefins $\mathbf{1 5}$ substituted with trifluoromethyl (15a-15b) and pinacolboryl (15d) groups, as well as in styrenes $(\mathbf{1 5 a}, \mathbf{1 5 c}, \mathbf{1 5 d}, \mathbf{1 5 e}, \mathbf{1 5 f})$, stilbenes, Michael acceptors (15e) and in alkyl-substituted alkenes. It also showed an excellent tolerance to functional groups including sulfides, tertiary amines, cyano, carboxylic acids, and alkynes. In addition, they also highlight that their 
protocol was able to cyclopropanate $\mathrm{E} / \mathrm{Z}$ styrene mixtures with absolute stereoconvergence (15f).

In comparison to our methodology, the Molander method shows a much broader scope. The Molander cyclopropanation permit the use of styrenes substituted in the $\alpha$ position with a boronic ester $\mathbf{1 5 d}$, a trifluoromethyl or a phenyl group $\mathbf{1 5} \mathbf{c}$ as well as $\alpha, \beta$ unsaturated esters 15e. Since the radical carbenoid is the same species in both cases, the distinct reactivity and broader scope might arise from the different redox environments under two distinct photoredox catalytic cycles. In fact, the mechanism proposed by Molander, Gutierrez and co-workers is in sharp contrast to ours, and rely on an anionic $\left(\mathrm{S}_{\mathrm{N}} 2\right)$ 3-exo-tet cyclization. The plausible mechanism, supported by experimental and extensive computational data is depicted in Scheme 3. Firstly, irradiation of 4CzIPN with visible-light generates its excited state $\mathbf{1 7}$, able to induce a single-electron oxidation with silicate $\mathbf{1 3}$ and form iodomethyl radical 6 . This reductive quenching of $4 \mathrm{CzIPN}^{*}$ is supported by Stern-Volmer emission quenching experiments and low oxidation potential of silicates $\left(E_{1 / 2}=+0.4-0.7 \mathrm{vs} \mathrm{SCE}\right)$. Addition of 6 to the corresponding alkene leads to a new radical intermediate 19. The latter species is reduce by $(-\cdot)[4 \mathrm{CzIPN}] \mathbf{1 8}$, forming anion 19' that furnishes the corresponding cyclopropane by an $S_{N} 2$ cyclization. In addition, the origin of the stereoconvergence for $\mathrm{E} / \mathrm{Z}$ styrene mixtures has been rationalized based on a similar argument to our hypothesis described in Scheme 3. With the difference that a stereoretentive reduction occurs prior to ring closure event. Alternatively, the authors also suggested a dynamic kinetic resolution-type scenario through a photochemical isomerization of the starting alkenes.

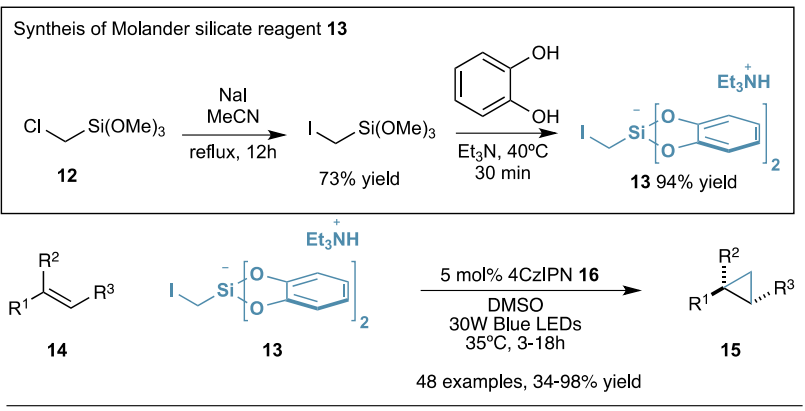

- selected examples

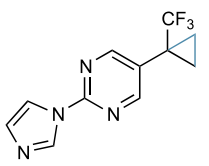<smiles></smiles><smiles>c1ccc(C2(c3ccccc3)CC2)cc1</smiles>

15a $65 \%$ yield 15b $47 \%$ yield $15 \mathrm{c} 98 \%$ yield

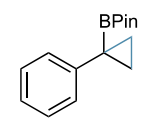<smiles>COC(=O)CC1CC1c1ccccc1</smiles>

$15 \mathrm{~d} 71 \%$ yield 15 e $65 \%$ yield

$\mathrm{Br}$<smiles>CC1CC1(C)c1ccc(Br)cc1</smiles>
$15 f 64 \%$ yield
from $E / Z 3: 1$

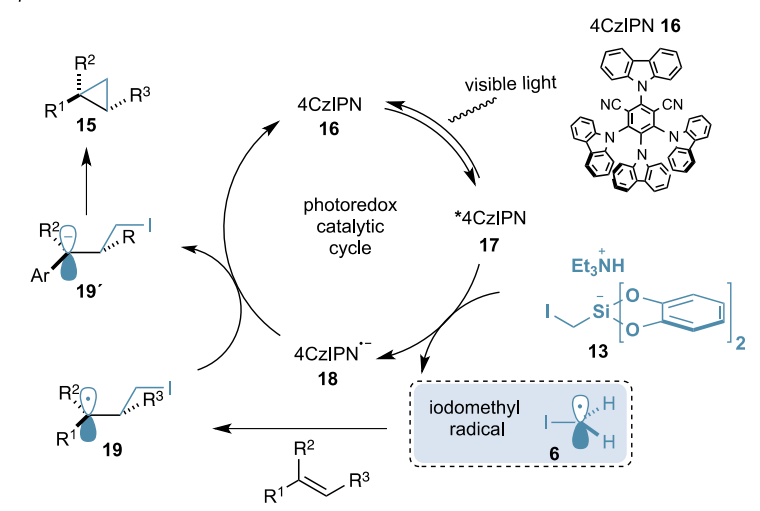

Scheme 5 Redox-neutral photocatalytic cyclopropanation via radical/polar crossover with iodomethylsilicate 13 by Molander et. al.

A clear experimental evidence that supports the anionic cyclization in the Molander cyclopropanation is shown in Scheme 6. When the cyclopropyl radical probe $\mathbf{2 0}$ was used as substrate, bis-cyclopropane $\mathbf{2 1}$ was obtained. This result differs from the outcome that we originally obtained with the analogous substrate 23, where ring opening product, via 25, was observed. Computational studies by Molander and Gutierrez showed that the ring-opening from the benzylic radical is much lower in energy than radical cyclization. Therefore, to explain formation of 21, it is proposed a fast SET reduction (to generate 22) that exceeds the rate of ring opening prior to ring formation. 
<smiles>C=C(C)c1ccccc1</smiles>
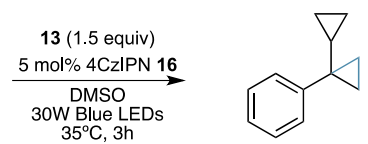

$2125 \%$ yield

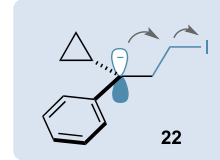<smiles>C=C(c1ccc(Br)cc1)C1CC1</smiles>

23

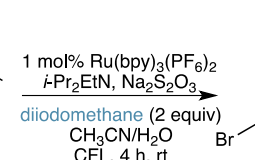
CFL, $4 \mathrm{~h}, \mathrm{rt}$

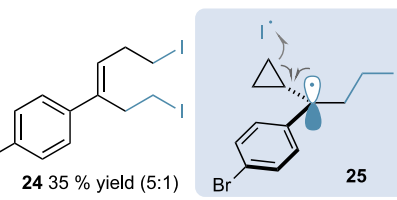
25
Scheme 6 Mechanistic experiments

After Molander's work, Li and co-workers reported an analogous cyclopropanation reaction using the well-known Ir-based photocatalyst $\operatorname{Ir}\left[\mathrm{dF}\left(\mathrm{CF}_{3}\right) \text { ppy }\right]_{2}(\mathrm{dtbbpy}) \mathrm{PF}_{6} \quad$ (30) instead of 4CzIPN and analogous reagent (27) that acts as a chloromethyl radical source (29) (Scheme 7). ${ }^{18}$ In contrast to the Molander process, Li showed a more modest diversity scope limited to electron-poor olefins $\mathbf{2 6}$ including $\alpha$-substituted phosphonates (28a, 28b), Michael acceptors (28c, 28d) and sulfones.

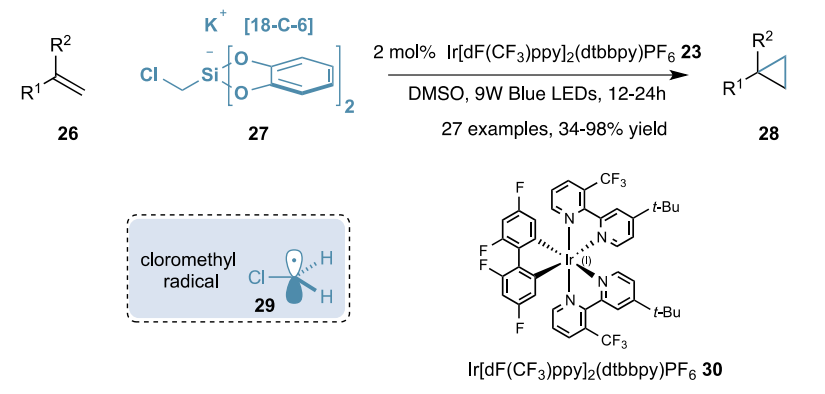

$$
\text { - selected examples }
$$
Scheme 7 Cyclopropanation reaction with chloromethylsilicate 27 by Li et. al.

Over the last three decades, the group of Charette has developed powerfull strategies for the diastereo- and enantioselective construction of cyclopropane rings based on the Simmons-Smith reaction with haloalkylzinc organometallics. ${ }^{19}$ In 2017, the group reported the synthesis of diiodomethylpinnacol boronate $\mathbf{3 2}$ as new reagent for the cyclopropanation of allyl ethers and styrenes via a Simmons-Smith reaction. ${ }^{20}$ After this, the authors realized about the suitability of this reagent as source of iodo(Bpin)methyl radical $\mathbf{3 5}$ enabled by photoredox catalysis and its application in the synthesis of borocyclopropanes of alkenes. $^{21}$ The authors demonstrated the first general borocyclopropanation of styrenes $\mathbf{3 1}$ using xanthone $\mathbf{3 3}$ as photocatalyst under continuous flow conditions and UVA-light (Scheme 8). The reaction was extensively explored in the styrene substrate, and worked well in a broad range of substrates substituted with alkyl chains, sufides, halides, ciano or nitro groups. The styrene substitution pattern showed to affect the efficiency of the reaction: whereas non-substituted styrenes or $\alpha, \alpha$-disubstituted styrenes worked well $(\mathbf{3 4 a}, \mathbf{c}, \mathbf{d}), \beta$-methyl
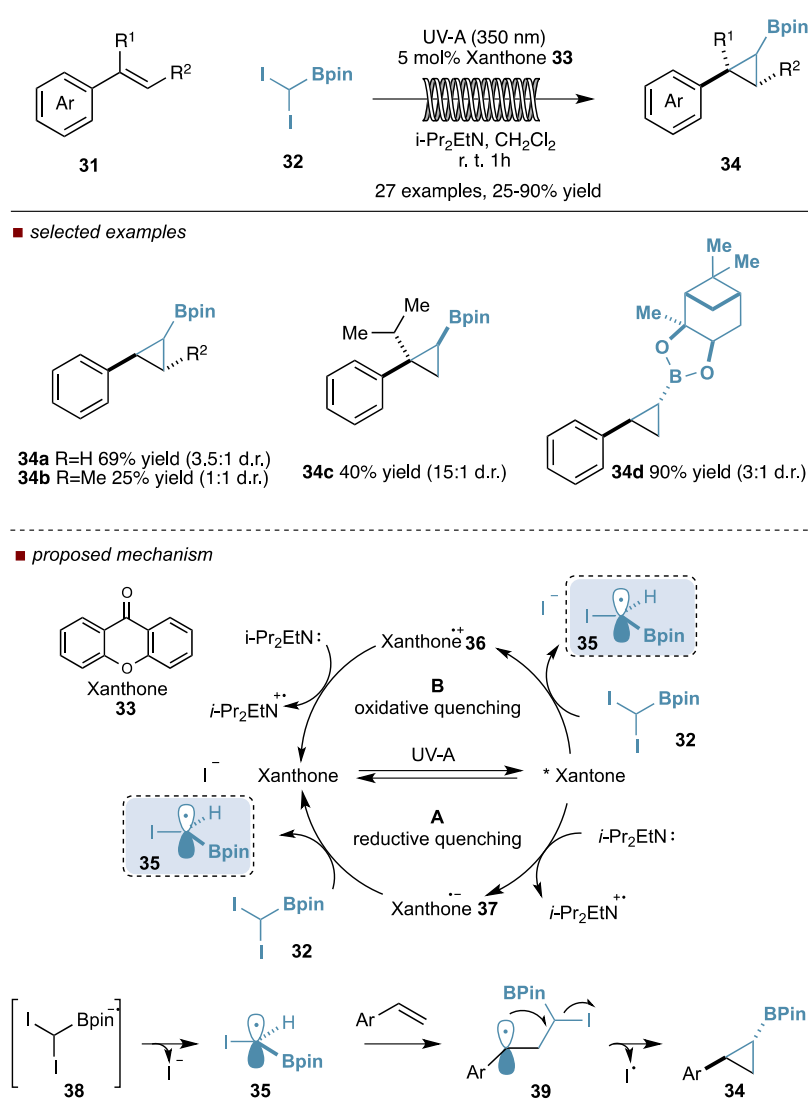

styrene provides low yield (34b, 25\%). The borocyclopropanes were obtained in moderate or low diastereoselectivity and the yields slightly drop after purification on chromatography column. However, the authors highlight that yields could be improved with sterically demanding boronates $(\mathbf{3 4 d}){ }^{22}$

Scheme 8 Borocyclopropanation mediated by UV-light under continuous flow conditions with diiodomethylpinnacol boronate 32 by Charette et. al.

Charette and co-workers postulated two possible mechanisms for the borocyclopropanation reaction of styrenes (Scheme 8). Initially, upon UVA-light irradiation, the corresponding xanthone* excited state can undergo both, reductive quenching (A) by $N, N$-diisopropylethylamine, or oxidative quenching (B) by boronate reagent 32 . However, the authors showed that the reductive quenching pathway (A) might be more favored, since (i) a significant excess of the base is used in comparison to $\mathbf{3 2}$, and (ii) it is kinetically favored based on Stern-Volmer fluorescent quenching studies $\left(k_{\mathrm{SV}}{ }^{i-\operatorname{Pr} 2 \mathrm{EtN}}=1.31 \times 10^{10} \mathrm{M}^{-1} \mathrm{~s}^{-1} ; k_{\mathrm{SV}}\right.$ ${ }_{\text {I2CHBpin }}=9.28 \times 10^{9} \mathrm{M}^{-1} \mathrm{~S}^{-1}$ ). Following the reductive quenching pathway, xanthone radical anion $\mathbf{3 7}$ could then undergo a SET process with $\mathbf{3 2}$ to form transient radical anion 38, which evolves into iodomethyl pinacol ester radical 35 upon fragmentation. After this, radical carbenoid $\mathbf{3 5}$ attacks the corresponding styrene and forms benzylic radical 39 that evolve to the borocyclopropane $\mathbf{3 4}$ by a radical 3-exo-tet cyclization.

Overall, the Charette method is a valuable contribution to the repertoire of the new photoredox cyclopropanations. The main advantage over Molander and our method is that it permits the 
construction of borocyclopropanes that can be further diversify by well-documented transformations.

Finally, a related radical cyclopropanation was reported by $\mathrm{Li}$, which in contrast to the previous methods, it requires the use of ethyl diazoacetate (EDA) as radical source (Scheme 9). ${ }^{23}$ In this work, a wide range of styrenes $\mathbf{4 0}$ were cyclopropanated and products $\mathbf{4 2}$ were obtained in good to excellent yields and with low diastereoselectivity using $\left[\mathrm{Ru}\left(\mathrm{bpy}_{3}\right)\right]^{2+} \mathbf{3}$ as photocatalyst. The tolerance of this reaction towards sensitive functional groups was not widely explored. In the mechanism, the authors proposed that excited Ru-polypyridine complex $\mathbf{4}$ is oxidized by $\mathbf{4 4}$ generating radical carbenoid $\mathbf{4 5}$. The latter species attacks the corresponding styrene and provide cyclopropane $\mathbf{4 2}$ and iodine radical by a radical 3-exo-tet cyclization. After this, iodine radical reacts with iodide to form anionic radical $\mathrm{I}_{2}(-\bullet)$. A second SET process closes the catalytic cycle ground state regenerating both ruthenium catalyst 3 and $I_{2}$. Interestingly, the scope of the reaction could be improved under thermal activation in the absence of a photocatalyst by heating EDA at $100{ }^{\circ} \mathrm{C}$ in the presence of $\mathrm{I}_{2}$. The authors proposed that ethyl diiodoacetate $\mathbf{4 4}$ is catalytically generated from EDA and $\mathrm{I}_{2} \cdot{ }^{24}$
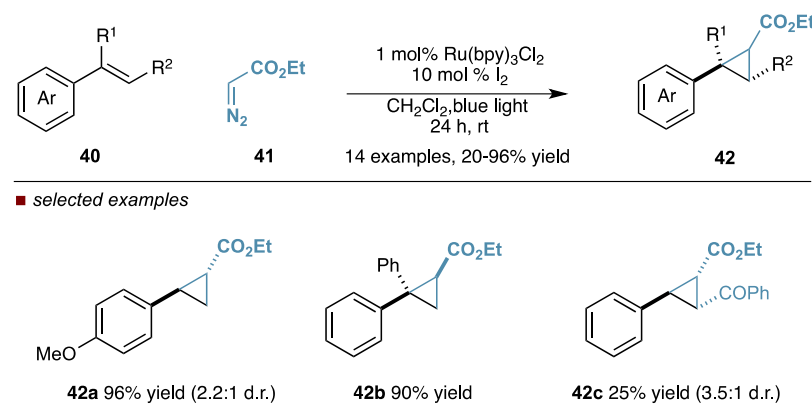

\section{- proposed mechanism}

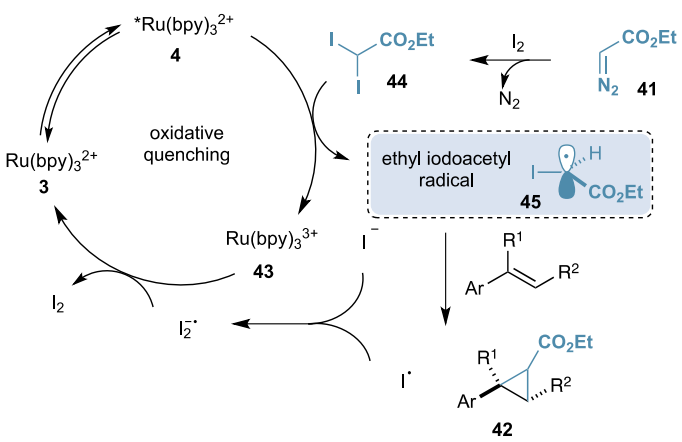

Scheme 9 lodine/photoredox-catalyzed cyclopropanation reaction with ethyl diazoacetate by Li et. al.

\section{Conclusions and outlook}

This Short-Review highlights the impact of photoredox catalysis in the discovery and development of new methodologies for cyclopropane synthesis involving radical carbenoids. The excellent functional group tolerance, mild reaction conditions and availability of the carbenoid sources are highly attractive features of these methods that may find immediate use in academic and industry laboratories. The development of a general methodology able to cyclopropanate olefins (activated and non-activated) with excellent diastereo- and enantioselectivity remains as one of the main challenges. Finally, we believe in the potential of electrochemistry to deliver, in the near future, a complementary approach for the cyclopropanation of alkenes based on the generation of radial carbenoids. ${ }^{25}$

\section{Acknowledgment}

The ICIQ Starting Career Programme, Agencia Estatal de Investigación of the Ministerio de Ciencia (CTQ2016-75311-P, AEI/FEDER-EU), the CELLEX Foundation through the CELLEX-ICIQ high-throughput experimentation platform and the CERCA Programme (Generalitat de Catalunya) are gratefully acknowledged for financial support. We thank the CELLEX Foundation for pre-doctoral fellowship (to A.G.H.). Dr Zhaofeng Wang is gratefully acknowledged for proofreading. M.G.S. is very grateful to the organizing committee of the 2018 Bürgenstock Conference and the Swiss Chemical Society for a JSP fellowship.

\section{References}

(1) (a) Chen, D. Y.-K.; Pouwer, R. H.; Richard, J. A. Chem. Soc. Rev. 2012, 41, 4631; (b) Salaün, J. Top. Curr. Chem. 2000, 207, 1.

(2) (a) Reissig, H.-U.; Zimmer, R. Chem. Rev. 2003, 103, 1151; (b) Schneider, T. F.; Kaschel, J.; Werz, D. B. Angew. Chemie Int. Ed. 2014, 53,5504 .

(3) Kulinkovich O. G. Cyclopropanes in Organic Synthesis, 2015, John Wiley \& Sons.

(4) Wessjohann, L. A.; Brandt, W.; Thiemann, T. Chem. Rev. 2003 103, 1625.

(5) For reviews on cyclopropanation reactions: (a) Lebel, H.; Marcoux, J. F.; Molinaro, C.; Charette, A. B. Chem. Rev. 2003, 103, 977. (b) Müller, P. Acc. Chem. Res. 2004, 37, 243. (c) Anilkumar, G. Biomolecular Chemistry. 2015, 13, 8780. (d) Qian, D.; Zhang, J. Chem. Rev. 2015, 44, 677. (d) Pons, A.; Poisson, T.; Pannecoucke, X.; Charette, A. B.; Jubault, P. Synthesis 2016, 48, 4060. (e) Trushkov, I. V. Isr. J. Chem. 2016, 56, 369. (f) Bos, D.; Poisson, T.; Pannecoucke, X.; Charette, A. B.; Jubault, P. Chem. Eur. J. 2017, 23, 4950. (g) Ebner, C.; Carreira, E. M. Chem. Rev. 2017, 117, 11651. (h) Wu, W.; Lin, Z.; Jiang, H. Org. Biomol. Chem. 2018, 16, 7315. For recent selected examples: (i) Coelho, P. S.; Brustard, E. M.; Kannan, A.; Arnold, F. H. Science 2013, 339, 307. (j) Künzi, S. A.; Manuel, J.; Toro, S.; Hartog, T. Den; Chen, P. Angew. Chem. Int. Ed. 2015, 54, 10670. (k) Bordeaux, M.; Tyagi, V.; Fasan, R. Angew. Chem. Int. Ed. 2015, 54, 1744; Angew. Chem. 2015, 127, 1764. (m) Pal, S.; Zhou, Y. Y.; Uyeda, C. J. Am. Chem. Soc. 2017, 139, 11686. (n) Pal, S.; Zhou, Y. Y.; Uyeda, C. J. Am. Chem. Soc. 2017, 139, 11686. (o) Werth, J.; Uyeda, C. Chem. Sci. 2018, 9, 1604. (p) Chen, K.; Zhang, S.-Q.; Brandenberg, 0. F.; Hong, X.; Arnold, F. H. J. Am. Chem. Soc. 2018, 140, 16402. (q) Jurberg, I. D.; Davies, H. M. L. Chem. Sci. 2018, 9, 5112. (r) Montesinos-Magraner, M.; Costantini, M.; RamírezContreras, R.; Muratore, M. E.; Johansson, M. J.; Mendoza, Angew. Chem. Int. Ed. 2019, 58, 5930.

(6) (a) Solorio-Alvarado, C. R.; Wang, Y.; Echavarren, A. M. J. Am. Chem. Soc. 2011, 133, 11952; (b) Vicente, R.; González, J.; Riesgo, L.; González, J.; López, L. A. Angew. Chem. Int. Ed. 2012, 51, 8063; Angew. Chem. 2012, 124, 8187; (c) Coelho, P. S.; Brustad, E. M.; Kannan, A.; Arnold, F. H. Science 2013, 339, 307; (d) Cotugno,P.; Monopoli, A.; Ciminale, F.; Milella, A.; Nacci, A. Angew. Chem. Int. Ed. 2014, 53, 13563; Angew. Chem. 2014, 126, 13781; (e) Mao, J.; Zhang, S.-Q.; Shi, B.-F.; Bao, W. Chem. Commun., 2014, 50, 3692; (f) Piou,T.; Rovis, T. J. Am. Chem. Soc. 2014, 136, 11292; (g) González, M. J.; González, J.; López, L. A.; Vicente, R. Angew. Chem. Int. Ed. 2015, 54, 12139; Angew. Chem. 2015, 127, 12307; (h) 
Manna, S.; Antonchick, A. P. Angew. Chem. Int. Ed. 2015, 54, 14845; Angew. Chem. 2015, 127, 15058; (i) Xu, G.; Renaud, P. Angew. Chem. Int. Ed. 2016, 55, 3657; Angew. Chem. 2016, 128, 3721; (j) Bajaj, P.; Sreenilayam, G.; Tyagi, V.; Fasan, R. Angew. Chem. Int. Ed. 2016, 55, 16110; Angew. Chem. 2016, 128, 26344; (k) Mato, M.; Herlé, B.; Holstein, P. M.; Echavarren, A. M. ACS Catal. 2017, 7, 3668. (l) Herlé, B.; Echavarren, A. M. Org. Lett. 2018, 20, 4341. (m) Jankins, T. C.; Fayzullin, R. R.; Khaskin, E. Organometallics 2018, 37, 2609; (n) Phipps, E. J. T.; Rovis, T. J. Am. Chem. Soc. 2019, 141, 6807.

(7) For recent reviews, see: (a) Narayanam, J. M. R.; Stephenson, C. R. J. Chem. Soc. Rev. 2011, 40, 102. (c) Prier, C. K.; Rankic, D. A.; MacMillan, D. W. C. Chem. Rev. 2013, 113, 5322. (d) Schultz, D. M.; Yoon, T. P. Science, 2014, 343, 1239176. (e) Romero, N. A.; Nicewicz, D. A. Chem. Rev. 2016, 116, 10075. (f) Wang, C.-S.; Dixneuf, P. H.; Soulé, J.-F. Chem. Rev. 2018, 118,7532 .

(8) (a) Nicewicz, D. A.; MacMillan, D. W. C. Science 2008, 322, 77; Narayanam, J. M. R.; Tucker, J. W.; Stephenson, C. R. J. J. Am. Chem. Soc. 2009, 131, 8756. (b) Nguyen, J. D.; D'Amato, E. M.; Narayanam, J. M. R.; Stephenson, C. R. J. Nat. Chem. 2012, 4, 854.

(9) (a) Beecraft, R. A.; Davidson, R. S. Goodwin, D. Tetrahedron Lett. 1983, 24, 5673; (b) Pandey, G. Photoinduced electron transfer (PET) in organic synthesis. Topics in Current Chemistry, 1993, 168, Springer, Berlin, Heidelberg; (c) Cossy, J.; Ranaivosata, J.-L.; Bellosta, V. Tetrahedron Lett. 1994, 35, 8161. (d) Cossy, J. Photoinduced Electron Transfer in Radical Reactions. Radicals in Organic Synthesis; John Wiley \& Sons, Ltd, 2008, 229.

(10) (a) Curran, D. P.; Chen, M.-H.; Kim, D. J. Am. Chem. Soc. 1986, 108, 2489; (b) Curran, D. P.; Chen, M.-H. Tetrahedron Lett. 1985, 26, 4991; (c) Byers, J. Atom Transfer Reactions. Radicals in Organic Synthesis; John Wiley \& Sons, Ltd, 2008, 72.

(11) The use of diiodomethane under UV-light irradiation for cyclopropanation reactions was firstly carried out by Simmons and Kropp; iodomethyl radical $(\bullet) \mathrm{CH}_{2} \mathrm{I}$ and iodomethyl cation $(+) \mathrm{CH}_{2} \mathrm{I}$ were proposed as reactive intermediates. (a) Blomstrom, D. E.; Herbig, K.; Simmons, H. E. J. Org. Chem. 1965, 30, 959. (b) Pienta, N. J.; Kropp, P. J. J. Am. Chem. Soc. 1978, 100, 655. (c) Kropp, P. J.; Pienta, N. J.; Sawyer, J. A.; Polniaszek, R. P. Tetrahedron, 1981, 37, 3229. (d) Kropp, P. J. Acc. Chem. Res. 1984, 17, 131. Later, density functional theory investigations performed by Phillips and co-workers suggested iso-diiodomethane $\left(\mathrm{CH}_{2}=\mathrm{I}-\mathrm{I}\right)$ as intermediate: (e) Phillips, D. L.; Fang, W. H.; Zheng, X. J. Am. Chem. Soc. 2001, 123, 4197.

(12) del Hoyo, A. M.; Herraiz, A. G.; Suero, M. G. Angew. Chemie Int Ed. 2017, 56, 1610

(13) (a) Curran, D. P.; Gabarda, A. E. Tetrahedron, 1999, 55, 3327. (b) Ohkita, T.; Tsuchiya, Y.; Togo, H. Tetrahedron, 2008, 64, 7247.

(14) del Hoyo, A. M.; Suero, M. G. Eur. J. Org. Chem. 2017, 2122.

(15) Nozaki, K.; Oshima, K.; Utimoto, K. Bull. Chem. Soc. Jpn. 1991, 64, 403.

(16) Phelan, J. P.; Lang, S. B.; Compton, J. S.; Kelly, C. B.; Dykstra, R.; Gutierrez, O.; Molander, G. A. J. Am. Chem. Soc. 2018, 140, 8037.

(17) Analogous chloromethyl and bromomethyl silicate reagents were also synthesized and tested. These reagents were not as efficient as the iodomethyl silicate reagent, and only in few substrates, the chloromethyl silicate reagent was competent. See ref 16 . for more details.
(18) Guo, T.; Zhang, L.; Liu, X.; Fang, Y.; Jin, X.; Yang, Y.; Li, Y.; Chen, B. Ouyang, M. Adv. Synth. Catal. 2018, 360, 4459.

(19) (a) Charette, A. B.; Cote, B.; Marcoux, J. F. J. Am. Chem. Soc. 1991, 113, 8166. (b) Charette, A. B.; Lemay, J. Angew. Chem. Int. Ed. 1997, 36, 1090. (c) Charette, A. B.; Juteau, H.; Lebel, H.; Molinaro, C. J. Am. Chem. Soc. 1998, 120, 11943.

(20) Benoit, G.; Charette, A. B. J. Am. Chem. Soc. 2017, 139, 1364.

(21) Sayes, M.; Benoit, G.; Charette, A. B. Angew. Chemie Int. Ed. 2018, 57, 13514.

(22) During the revision of this review, we realized of a related borocyclopropanation to Charette reaction: Ohtani, T.; Tsuchiya, Y.; Uraguchi, D.; Ooi, T. Org. Chem. Front. 2019. DOI: $10.1039 /$ c9qo00197b.

(23) Li, P.; Zhao, J.; Shi, L.; Wang, J.; Shi, X.; Li, F. Nat. Commun. 2018, 9, 1972.

(24) For alternative reports of alkene cyclopropanations enabled by photoredox catalysis that do not involve radical carbenoids: (a) Sarabia, F. J.; Ferreira, E. M. Org. Lett. 2017, 19, 2865. (b) Wang, Z.; Herraiz, A. G.; del Hoyo, A. M.; Suero, M. G. Nature 2018, 554, 86. (c) Zhang, Y.; Qian, R.; Zheng, X.; Zeng, Y.; Sun, J.; Chen, Y.; Ding, A.; Guo, H. Chem. Commun. 2015, 51. (d) Shu, C.; Mega, R. S.; Andreassen, B. J.; Noble, A.; Aggarwal, V. K. Angew. Chemie Int. Ed. 2018, 57, 15430.

(25) The use of electrochemistry for alkene cyclopropanation with gem-dihaloalkanes has been demonstrated scarcely in the past and the involvement of halomethyl radicals was proposed: (a) Léonel, E.; Dolhem, E.; Devaud, M.; Paugam, J. P.; Nédélec, J. Y. Electrochim. Acta, 1997, 42, 2125; (b) Njue, C. K.; Nuthakki, B.; Vaze, A.; Bobbitt, J. M.; Rusling, J. F. Electrochem. commun. 2001, 3, 733.

\section{Biosketches}




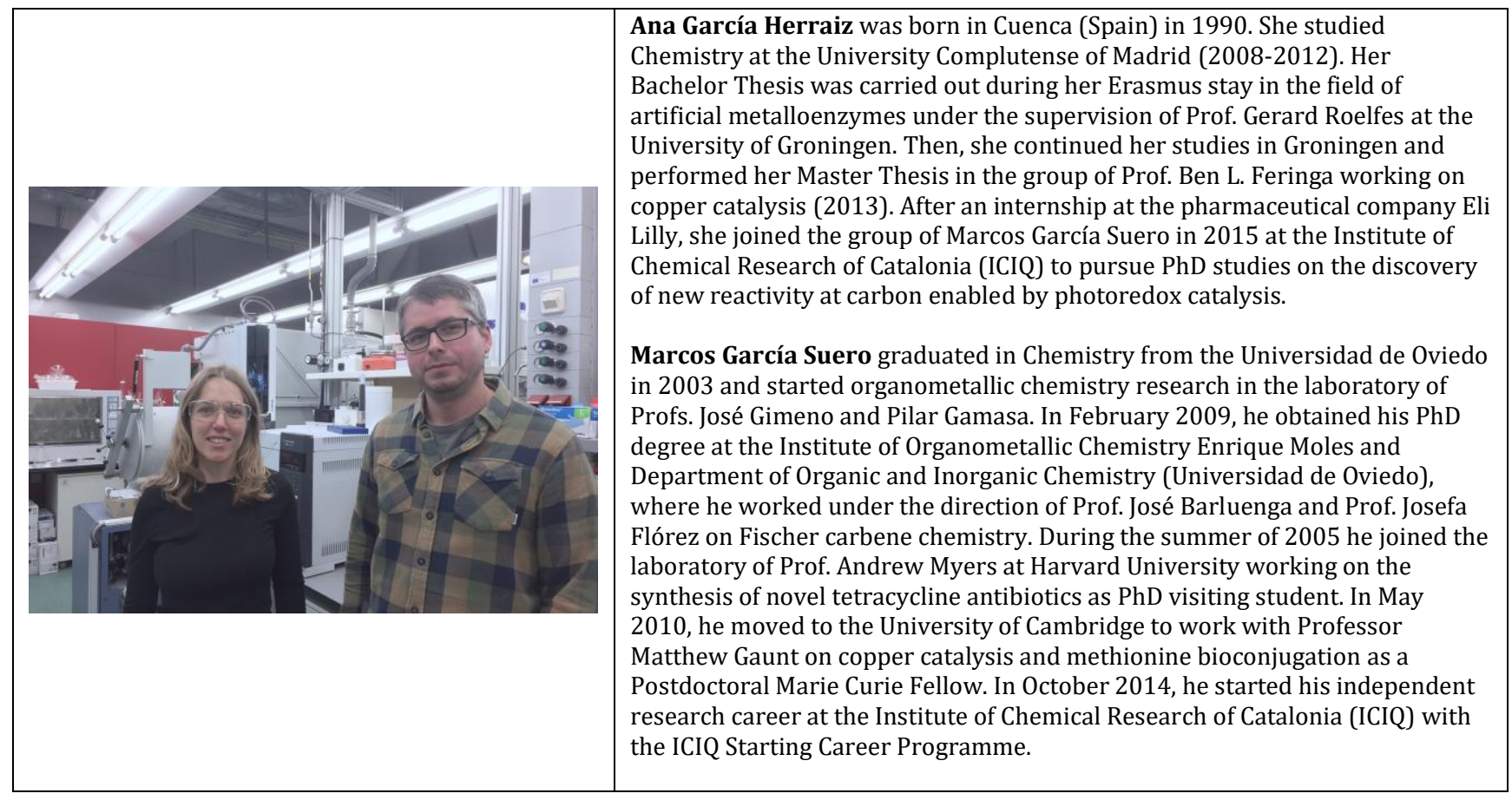

